\title{
Live recombinant Lactococcus lactis vaccine expressing aerolysin genes D1 and D4 for protection against Aeromonas hydrophila in tilapia (Oreochromis niloticus)
}

\begin{abstract}
Aims: To evaluate a live recombinant Lactococcus lactis vaccine expressing aerolysin genes D1 (Lac-D1ae) and/or D4 (Lac-D4ae) in protection against Aeromonas hydrophila in tilapia (Oreochromis niloticus). Methods and Results: The polymerase chain reaction (PCR)-amplified 250- and 750-bp sequences coding for domains D1 and D4 of aerolysin were individually cloned into pNZ8048 and electrotransformed into L. lactis. The recombinant vaccine candidates were then either orally fed or injected intraperitoneally into tilapia. The development of antibodies in sampled fish compared to control groups implied that the recombinant epitopes expressed in L. lactis were able to elicit an immunogenic response in tilapia. Interestingly, the lower doses of both Lac-D1ae and Lac-D4ae gave higher antibody levels over the study period. Fish immunized with Lac-D1ae and Lac-D4ae together showed the highest level of protection, and the mortality was reduced significantly compared to control strains in both modes of vaccination. Conclusions: The recombinant L. lactis strain expressing D1 and D4 produced aerolysin-specific serum IgM in tilapia. Both D1 and D4 promoted 55-82\% relative per cent survival (RPS) against Aeromonas infection through intraperitoneal injection, whereas the RPS following oral feeding of the vaccine was 70-100\%. Significance and Impact of the Study: The D1 and D4 regions of the aerolysin protein have been successfully identified as immunogenic regions that can elicit antibody production in tilapia and protect against challenge with Aer. hydrophila. A promising oral vaccine using L. lactis harbouring the D1 and D4 regions has been developed to control Aer. hydrophila.
\end{abstract}

Keyword: Aerolysin; Aeromonas hydrophila; Lactococcus lactis; Recombinant vaccine; Tilapia 